\title{
Cáncer en la pandemia por COVID-19
}

\section{Cancer in the COVID-19 pandemic}

\author{
Jesús Solier Insuasty-Enríquez, Jenny Paola Garzón-Hernández • Bucaramanga (Colombia)
}

DOI: https://doi.org/10.36104/amc.2020.1916

La pandemia de enfermedad por el virus SARS-CoV-2 (COVID-19) ha representado que alrededor del mundo millones de personas se encuentren en estado de aislamiento social o en cuarentena preventiva. Son mas de 33.7 millones de casos confirmados y aproximadamente 1010381 casos fatales, según los recientes datos de la Universidad Johns Hopkins. En Colombia se han reportado a la fecha (septiembre 30) 829679 casos (con un pico importante de 5637 casos nuevos en el ultimo día) y 25998 defunciones (1). En Italia de acuerdo con los estudios publicados, se estimó que alrededor del $20 \%$ de los pacientes con COVID-19 tenían cáncer (2). Estados Unidos, un estudio de cohortes retrospectivo con la muestra mas grande de 928 pacientes adultos hasta el momento con cáncer activo o previo y con síndrome respiratorio agudo grave por COVID-19, captados de EE. UU., Canadá y España [Cancer Consortium (CCC19)], entre el 17 de marzo y el 16 de abril de 2020 se evidencia que, la mediana de edad fue de 66 años, un 30\% mayores de 75 años y de los cuales $50 \%$ eran hombres. Los cánceres más prevalentes fueron cáncer de mama (191 [21\%]) y cáncer de próstata (152 [16\%]). En tratamiento activo se encontraban 366 (39\%) pacientes de los cuales sólo 121 (13\%) pacientes habían muerto. En el análisis de regresión logística, los factores independientes asociados con el aumento de la mortalidad a los 30 días, fueron: edad avanzada (por 10 años; odds ratio parcialmente ajustado 1.84, IC 95\% 1.53-2.21), sexo masculino (1.63, 1.07-2.48), tabaquismo (ex fumador versus nunca había fumado: 1.60, 1.03-2.47), número de comorbilidades (dos versus ninguno: 4.50, 1.33-15.28), estado de desempeño del Eastern Cooperative Oncology Group con un puntaje de 2 o más (estado de 2 vs 0 o 1:3. 89, 2.11-7.18), un cáncer activo (progresivo vs remisión: 5.20, 2.77-9.77), y tratamiento con azitromicina más hidroxicloroquina (versus tratamiento con ninguno: 2.93, 1.79-4.79). La raza y el origen étnico, el estado de obesidad, el tipo de cáncer y de tratamiento oncológico, y la cirugía reciente no se asociaron con una mayor tasa de mortalidad (3).

El metaanálisis más completo realizado hasta la fecha de elaboración de este manuscrito, acumuló datos de 32 estudios con 46499 pacientes (1776 pacientes con cáncer) con COVID-19 de Asia, Europa y los Estados Unidos, cuantificó el efecto del cáncer con resultados clínicos importantes, como la tasa de mortalidad y la necesidad de admisión a las Unidades de Cuidados Intensivos (UCI), encontraron que los pacientes de edad avanzada con cáncer, pueden no tener un mayor riesgo de muerte incluso durante la infección por la enfermedad COVID-19. Los hallazgos de este estudio son importantes y motivan a continuar en la investigación para obtener conclusiones objetivas que puedan orientar las políticas de salud pública en la protección de la población de pacientes oncológicos (4).

Mientras las investigaciones maduran se debe considerar a los pacientes con cáncer, como individuos de gran vulnerabilidad para cualquier tipo de infección, debido a su estado de inmunosupresión secundaria a su enfermedad subyacente, con un mayor riesgo de desarrollar el síndrome respiratorio agudo grave por COVID-19, ingresar a UCI e incluso fallecer a consecuencia de esta mortal infección (3). Así mismo, el paciente con cáncer, es un paciente que podría verse afectado en los casos de retrasos en el tratamiento por la dificultad al recibir oportunamente las terapias esenciales o que se enfrenten a la escasez
Dr. Jesús Solier Insuasty-Enríquez: Internista, Oncólogo-Clínico. Director del grupo de Investigación Germina-UIS. Profesor Departamento de Medicina Interna Universidad Industrial de Santander; Oncólogo-Clínico Unidad de Oncología Hospital Universitario de Santander e Insuasty Oncología e Investigación S.A.S.; Jenny Paola Garzón-Hernández: Estudiante de Medicina. Auxiliar de Investigación grupo GERMINA, Departamento de Medicina Interna Universidad Industrial de Santander. Bucaramanga (Colombia).

Correspondencia: Dr. Jesús Solier InsuastyEnríquez. Bucaramanga (Colombia). E-mail: jesusinsuastyasco@hotmail.com Recibido:19/VI/2020 Aceptado: 05/X/2020 
de insumos y medicamentos durante el estado de emergencia sanitaria global (5).

Si bien la evidencia es limitada, las sociedades científicas como la American Association of Clinical Oncology (ASCO), European Bone Marrow Transplantation (EBMT), la American Society for Transplantation and cellular therapy (ASTCT) y en nuestro pais, la Asociación Colombiana de Oncología y Hematología (ACHO) han publicado recomendaciones sobre cómo modificar la práctica clínica durante la atención, tratamiento y seguimiento de los pacientes con cáncer durante estas circunstancias excepcionales, buscando reducir las visitas innecesarias a los hospitales y considerar opciones como las plataformas de telemedicina, por mencionar algunas como medios de protección para lo pacientes y/o sus familias (6). Ahora, las nuevas tecnologías, como la tele-oncología, han demostrado ser una alternativa eficiente en el seguimiento y supervisión de los esquemas de quimioterapia y radioterapia, ser una herramienta educativa para el paciente y/o cuidador y para el oncólogo clínico en su actualización continua, en la investigación y la colaboración interdisciplinar entre expertos (7).

Aunque por el momento no es posible conocer el real impacto de la pandemia sobre el cáncer en nuestro país, una alta mortalidad podría estar asociada a los factores de riesgo generales ya conocidos y a factores de riesgo específicos en los pacientes con cáncer. Es por esto que se adelanta un estudio nacional liderado por la ACHO que permitirá dar luces del comportamiento, las características y desenlaces en nuestros pacientes oncológicos durante la pandemia por COVID-19. Por ahora los servicios de oncología continuarán adaptándose y aprendiendo de las experiencias de los países altamente afectados por esta infección, para continuar garantizando el mejor cuidado de los pacientes, en el sentido de no olvidar a ninguno de ellos.

\section{Referencias}

1. OPS. Reporte de Situación No 151-30 de septiembre de 2020. https://www.paho. org/es/documentos/reporte-situacion-covid-19-colombia-no-151-30-septiembrede-2020 [Internet]. 2020;1(151):1-9.

2. Onder G, Rezza G, Brusaferro S. Case-fatality rate and characteristics of patients dying in relation to COVID-19 in Italy. JAMA. 2020, doi: 10.1001/jama.2020.4683.

3. Kuderer NM, Choueiri TK, Shah DP, Shyr Y, Rubinstein SM, Rivera DR, et al. Clinical impact of COVID-19 on patients with cancer (CCC19): a cohort study. Lancet 2020;6736(20):1-13.

4. Giannakoulis VG., Papoutsi E.1, Siempo EI. Effect of Cancer on Clinical Outcomes of Patients With COVID-19: A Meta-Analysis of Patient Data. JCO Glob Oncol. 2020 junio; 6: 799-808

5. Yu J, Ouyang W, Chua ML, Xie C. SARS-CoV-2 transmission in patients with cancer at a tertiary care hospital in Wuhan, China. JAMA Oncol. 2020

6. Hematolog CDE. Recomendaciones ACHO para el manejo de estado de Pandemia SARS - COV 19 Recomendaciones para los Servicios de salud que atienden pacientes hematológicos y / o con Cáncer. Recomendaciones para los especialistas en el manejo de los pacientes con Cáncer y enfermedades Hematólogicas . Siempre tener en cuenta que : 2020;(123).

7. Sirintrapun S.J., Lopez A.M. Telemedicine in cancer care. Am. Soc.Clin. Oncol . Educ. Book. 2018;38:540-545. 